\title{
Conduite des troupeaux allaitants en zones difficiles
}

\author{
M Thériez, M Petit, W Martin-Rosset \\ INRA, centre de Clermont-Theix, département Élevage et Nutrition des herbivores, \\ 63122 Saint-Genès-Champanelle, France
}

Les troupeaux de bovins, d'ovins et d'équidés, élevés en milieux difficiles, sont soumis à différentes contraintes, dont la principale est une période de faible disponibilité alimentaire de durée plus ou moins longue. Au cours de celle-ci, les animaux en stabulation sont souvent alimentés avec des fourrages de qualité médiocre à mauvaise, récoltés à un stade de maturité trop tardif. Ce type de régime entraîne des déficits alimentaires plus ou moins marqués, selon la capacité de l'espèce à ingérer des aliments à forte valeur d'encombrement. Au pâturage, les différences entre espèces sont moins marquées, du fait des possibilités de tri. Cette période difficile entraîne une mobilisation des réserves corporelles, dont l'intensité dépend de la durée de la sousalimentation, de l'état corporel initial, et surtout du stade physiologique de la femelle, c'est-à-dire de ses besoins nutritionnels. Dans les 3 espèces étudiées, la productivité des troupeaux étant conditionnée par le niveau nutritionnel et par l'état corporel des femelles au moment de la mise bas et de la mise à la reproduction, il est nécessaire d'adapter les méthodes d'élevage pour permettre aux animaux de ne pas descendre en dessous d'états minima.

Ces adaptations portent sur le type de production (le plus souvent des jeunes qui sont vendus pour être engraissés dans des zones plus favorables), le choix des races et les systèmes de conduite. Les races adaptées à ces milieux se caractérisent par leur capacité à ingérer suffisamment de fourrages grossiers et par leur aptitude à utiliser et à reconstituer leurs réserves corporelles. Cela leur confère une capacité satisfaisante à produire régulièrement des jeunes et à les élever. La brièveté de la période d'alimentation abondante au pâturage limitant les possibilités de reconstitution des réserves corporelles, la première adaptation des systèmes de conduite aux conditions difficiles consiste à choisir des dates de reproduction et de mise bas qui permettent de faire coincider ces périodes de besoins élevés avec celle de disponibilité alimentaire importante. La durée d'allaitement peut aussi être raccourcie. 